\title{
Nanoscale Quantification of Jahn-Teller Distortion in $\mathrm{LaMnO}_{3}$
}

\author{
Binbin Wang ${ }^{1,2}$, Bryan D. Esser ${ }^{1}$, Nuria Bagués ${ }^{1}$, Jiaqiang Yan $^{3}$ and David W. McComb ${ }^{1,2 *}$ \\ 1. Center for Electron Microscopy and Analysis, The Ohio State University, Columbus, OH USA. \\ 2. Dept. of Materials Science \& Engineering, The Ohio State University, Columbus, OH, USA \\ 3. Materials Science and Technology Division, Oak Ridge National Laboratory, TN 37831, USA \\ * Corresponding author: mccomb.29@osu.edu
}

Cooperative Jahn-Teller distortions (CJTD), which appear in the octahedral sites of some transition metal ions (i.e. $\mathrm{Cu}^{2+}$ and $\mathrm{Mn}^{3+}$ ), is a phenomenon related to many interesting physical properties, including charge ordering, spin ordering and orbital ordering [1, 2]. For example, in $\mathrm{LaMnO}_{3}$, the electron-phonon interaction causes the Jahn-Teller splitting of $\mathrm{e}_{\mathrm{g}}$ levels, where a Q2 octahedral distortion is typically observed: two Mn-O bonds shorten, and two Mn-O bonds lengthen [3].

Experimentally, a precise determination of the octahedral bonding parameters in oxides can be achieved by Rietveld refinement of neutron and X-ray diffraction techniques [4-6]. However, these techniques probe volume averaged properties of materials. In order to study the local properties of materials, such as interface coupling of octahedral connectivity in perovskite oxide heterostructures and the topological hall effects driven by octahedral tilting in ultrathin films aberration corrected scanning transmission electron microscopy (STEM) offers the highest spatial resolution [7, 8].

Due to the relatively weak scattering cross-section of oxygen $(\mathrm{O})$, techniques such as annular bright field (ABF) imaging, electron energy loss spectra (EELS) and differential phase contrast (DPC) imaging are necessary to image the $O$ columns in real space [9-11]. However, rotation of the octahedra in complex oxides can make it challenging to image $\mathrm{O}$ columns precisely and potential image distortion caused by probe instabilities and sample drift can further complicate the situation. For example, as shown in Fig.1a, certain oxygen sites are co-aligned with some of the heavy atom positions in the [110] projection. While post-processing procedures can allow the octahedral rotation types to be deduced in certain cases, the magnitude of the bond-distance changes in the oxygen octahedra is a challenge in even the most advanced STEM instruments. For example, Mn-O bond lengths in $\mathrm{LaMnO}_{3}$ differ by 6-27 pm due to CJTD; whereas, without considering CJTD, they only differ by approximately $7 \mathrm{pm}$.

An alternative technique to investigate the structural origins of the properties of nanoscale perovskites with distorted octahedra is position-averaged convergent beam electron diffraction (PACBED), which has been proven to be sensitive to structural information, such as thickness, distortion and polarity [12]. As shown in Fig.1b, the simulated PACBED is in good agreement with experimental results for a sample thickness of approximately $27 \mathrm{~nm}$. By comparing experimental and simulated results, octahedral rotation in perovskites can be quantitatively measured [13].

In this work, we propose an approach to precisely detect the magnitude of CJTD using PACBED. The picometer-size CJTD-introduced change in bond distance in $\mathrm{LaMnO}_{3}$ can be detected experimentally and verified by simulation. In Fig.2, the minimum normalized $X^{2}$ shows the best agreement between simulated PACBED and experimentally derived values [4] when considering a combination of octahedral rotations at different bonding ratios ( $\mathrm{Mn}-\mathrm{O} 2$ (short)/Mn-O2(long)). The optimized fit appears when the bonding ratio is the closest to the experimental values, as indicated by the arrow in Fig.2a. These data show that octahedral distortions due to CJTD are experimentally detectable when verified by simulation, helping to 
investigate local CJTD-related distortions and their effect on physical properties.

References:

[1] B Keimer, Nature materials 5 (2006), p. 933.

[2] Y Tokura and N Nagaosa, science 288 (2000), p. 462.

[3] MW Lufaso and PM Woodward, Acta Crystallogr B 60 (2004), p. 10.

[4] J Rodriguez-Carvajal et al., Physical Review B 57 (1998), p. R3189.

[5] J Song et al., Physical Review B 66 (2002), p. 020407.

[6] S May et al., Physical Review B 82 (2010), p. 014110.

[7] L Qiao et al., Nano letters 15 (2015), p. 4677.

[8] Y Gu et al., arXiv preprint arXiv:181109075, (2018), p.

[9] AY Borisevich et al., Physical review letters 105 (2010), p. 087204.

[10] R Aso et al., Sci Rep 3 (2013), p. 2214.

[11] N Shibata et al., Nature communications 8 (2017), p. 15631.

[12] JM LeBeau et al., Ultramicroscopy 110 (2010), p. 118.

[13] J Hwang et al., Applied Physics Letters 100 (2012), p.

[14] Funding for this research was provided by the Center for Emergent Materials at the Ohio State University, an NSF MRSEC (Award Number DMR-1420451).
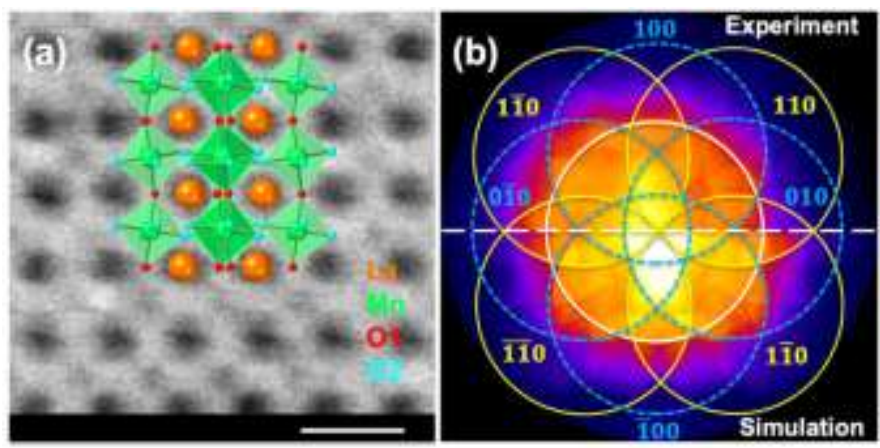

Figure 1. (a) Bright field STEM image of $\mathrm{LaMnO}_{3}$ along the [110] after non-rigid registration with Pbnm crystal model overlay. Scale bar is $1 \mathrm{~nm}$. (b) Comparison of experimentally measured and simulated PACBED of the $\mathrm{LaMnO}_{3}$ [001] with $27 \mathrm{~nm}$ thickness.
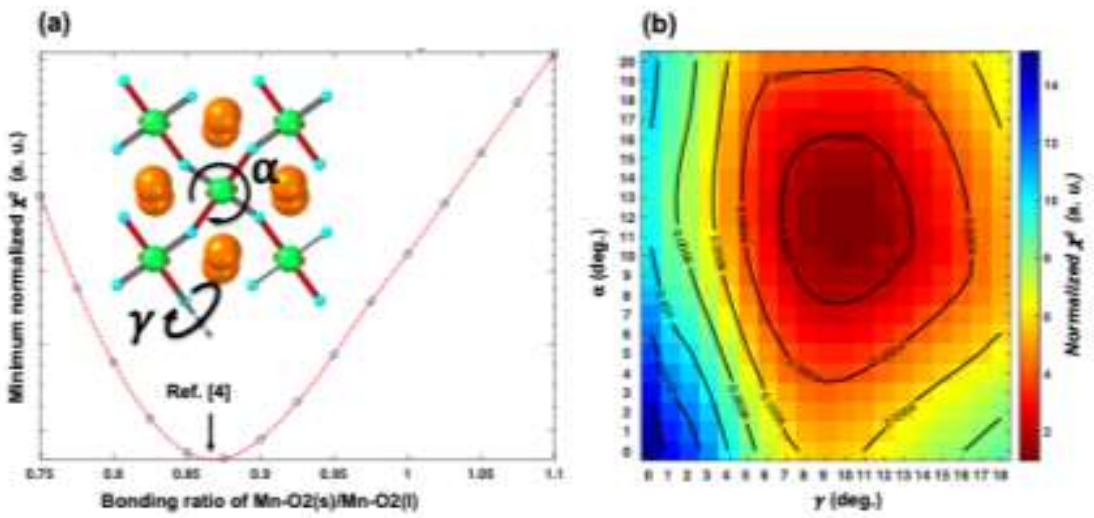

Figure 2. Quantification of the effects of (a) Jahn-Teller distortions and (b) octahedral tilts in simulated PACBED of $\mathrm{LaMnO}_{3}$. 